\title{
Editorial
}

\section{Work Counts - So Count It}

\author{
Gregory R. Wagner \\ National Institute for Occupational Safety and Health, Washington, D.C., USA
}

Public health surveillance involves the ongoing collection, analysis, evaluation, and reporting of health-relevant information to improve prevention. Surveillance of occupational health, like other public health endeavors, has been a powerful tool for tracking trends, evaluating the success or failure of interventions, and identifying new problems or the new circumstances where wellknown problems are arising. Surveillance data can provide a measure of the extent of a problem and be invaluable in the allocation of scarce prevention resources [1].

Surveillance systems often rely on information collected in administrative systems such as death certificates or hospital discharge diagnoses. Alternatively, surveillance can be active and case-based, depending on the recognition and reporting of particular conditions by health care providers or by a subset of volunteers. For example, in the UK, the Health and Occupation Research Network (THOR) system has provided useful information about trends in work-related and occupational lung diseases by engaging voluntary reporters from amongst the specialists in respiratory or occupational medicine [2].

In this issue of Respiration, Amar et al. [3] demonstrate both the feasibility and the value of analyzing data from the electronic medical records of general practitioners in order to better understand the incidence and temporal trends of the pneumoconioses in the UK. From the medical records of approximately 5 million patients felt to be representative of the UK population as a whole, the authors identified 1,070 incident cases of pneumoconiosis

\section{KARGER}

for the years $1997-2008$. Of these cases, $87 \%$ were asbestosis. From this, they estimate that there are approximately 1,800 new cases of asbestosis and an additional 600 cases of non-asbestos-related pneumoconiosis per year. This compares with the total of 637 cases of pneumoconiosis reported by specialists in the THOR system between 1999 and 2006, or approximately 80 cases per year [2].

This difference between the reports, while large, is not particularly surprising. General practitioners see all who seek care. Specialists generally see patients on referral; only the more challenging or difficult cases are likely to be referred to them.

What is surprising, however, is the absence of information about the work of the people identified with incident occupational lung diseases by this study. While it is of great interest and benefit to have national pneumoconiosis incidence estimates, it would also be beneficial to understand incidence rates in the sector of the population which is actually at risk. The pneumoconioses are almost exclusively occupational in etiology, almost always resulting from workplace exposure to asbestos, silica or coal mine dust. The risk of disease for those who have been exposed to hazardous dust and have lived through the latency period is much greater than the risk to the population as a whole.

The views expressed are those of the author and not necessarily those of the National Institute for Occupational Safety and Health. 
An individual's work matters, both for understanding the data and when planning medical interventions. Unfortunately, there is nothing in the analysis that considers the industry or occupation of the patients with incident pneumoconiosis. This is most likely because the relevant information was not available for analysis, either because (as is commonly the case) it had not been elicited or perhaps because it is was just not included in the electronically-coded component of the medical records. Medical records generally include little or no work-related information, despite the fact that adults who work full-time spend one third of their waking lives at work.

\section{Why Should We Care?}

First, information about industry and occupation could help target limited preventive resources. Despite the latency between first exposure and manifestation of disease, there may be persistent conditions of excessive exposure in a patient's current or former workplace which merit control or monitoring [4]. It would also be useful to know, for example, if asbestosis or silicosis occurs in peo- ple who have not worked in industries where exposures to asbestos or silica are common.

Furthermore, knowing the distribution of disease according to occupation can help clinical decision-making. People with asbestos exposure are at increased risk for lung cancer. Those with asbestosis are only the tip of the iceberg of the exposed population; therefore, enhanced health education and surveillance and possibly lung cancer screening is warranted in asbestos-exposed workers. Information about the industry and occupation of newlydiagnosed patients may identify other occupational groups who are not expected to be at risk.

The routine inclusion of occupational information in electronic health records is under discussion in the USA. A recent report from the US National Academies of Science notes, 'Incorporating patients' occupational information into electronic health records could lead to more informed clinical diagnosis and treatment plans as well as more effective policies, interventions, and prevention strategies to improve the overall health of the working population' [5]. The informative report from Amar et al. [3] would have been even more valuable had information about work been readily available.

\section{References}

1 Wagner GR, Fine LJ: Surveillance and health screening in occupational health; in Wallace (ed): Public Health and Preventive Medicine, ed 15. New York, McGraw-Hill, 2008, pp 759-793.

- 2 Turner S, McNamee R, Carder M, Agius R: Trends in pneumoconiosis and other lung diseases, as reported to a UK-based surveillance scheme for work-related ill health. J Phys Conference Series 2009;151:1-6.
Amar RK, Jick SS, Rosenberg D, Maher TM, Meier CR: Incidence of the pneumoconioses in the United Kingdom general population between 1997 and 2008. Respiration 2012;84:200-206.
Valiante DJ, Rosenman KD: Does silicosis still occur? JAMA 1989;262:3003-3007.

5 Wegman DH, Liverman CT, Schultz AM, Strawbridge LM (eds): Incorporating Occupational Information in Electronic Health Records: Letter Report Institute of Medicine, Washington DC, 2011. 